\title{
Relationship between diffusion capacity and small airway abnormality in COPDGene
}

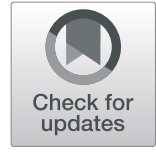

\author{
Rachel N. Criner ${ }^{1 *}$ D, Charles R. Hatt ${ }^{2,3}$, Craig J. Galbán³ , Ella A. Kazerooni ${ }^{3}$, David A. Lynch ${ }^{4}$, \\ Meredith C. McCormack ${ }^{5}$, Richard Casaburi ${ }^{6}$, Neil R. Maclntyre ${ }^{7}$, Barry J. Make ${ }^{8}$, Fernando J. Martinez ${ }^{9}$, \\ Wassim W. Labaki ${ }^{10}$, Jeffrey L. Curtis ${ }^{10,11}$ and Mei Lan K. Han ${ }^{10}$
}

\begin{abstract}
Impaired single breath carbon monoxide diffusing capacity (DLCO) is associated with emphysema. Small airways disease (SAD) may be a precursor lesion to emphysema, but the relationship between SAD and DLCO is undescribed. We hypothesized that in mild COPD, functional SAD (FSAD) defined by computed tomography (CT) and Parametric Response Mapping methodology would correlate with impaired DLCO. Using data from eversmokers in the COPDGene cohort, we established that fSAD correlated significantly with lower DLCO among both non-obstructed and GOLD 1-2 subjects. The relationship between DLCO with CT-defined emphysema was present in all GOLD stages, but most prominent in severe disease.
\end{abstract}

Trial registration: NCT00608764. Registry: COPDGene. Registered 06 February 2008, retrospectively registered.

Keywords: Diffusing capacity of the lung (DLCO), Small airways disease (SAD), Chronic obstructive pulmonary disease (COPD), Parametric response mapping (PRM)

\section{Introduction}

The importance of small airways disease (SAD) in COPD pathogenesis was first described by Hogg et al. in the 1960s, who determined that peripheral resistance contributes minimally to total airway resistance in healthy lungs but significantly in emphysematous lungs [1]. Recent research using advanced computed tomography $(\mathrm{CT})$ has further suggested that a CT metric for small airway disease (SAD) radiographically precedes emphysema development and is associated with subsequent $\mathrm{FEV}_{1}$ decline $[2,3]$.

Diffusing capacity of the lung (DLCO) is a commonly tested measurement in pulmonary function studies, indirectly measuring degree of gas transfer from alveoli to pulmonary capillary blood, with results depending on the lung's structural and functional properties. Emphysema irreversibly destroys alveoli, leading to gas exchange impairment and manifesting as an inverse relationship between emphysema severity and DLCO [4]. We hypothesized that CT defined SAD among

\footnotetext{
* Correspondence: rachel.criner@pennmedicine.upenn.edu

${ }^{1}$ Division of Pulmonary, Allergy and Critical Care, University of Pennsylvania, 839 West Gates Building, Philadelphia, PA 19104, USA

Full list of author information is available at the end of the article
}

COPD subjects with mild disease (GOLD 1-2) may detect lung regions in transition from bronchiolar pathology to emphysema, and hence, may be associated with diffusion capacity impairment.

\section{Methods}

We analyzed data from the five-year follow-up visit of the COPDGene cohort in individuals $(n=1846)$ with $\geq 10$ pack-years smoking history and GOLD spirometric grades $0-4$ for whom DLCO and CT imaging data were available and obtained on the same day. GOLD 0, although no longer used in the GOLD strategic document, includes non-obstructed smokers (post-bronchodilator $\mathrm{FEV}_{1} / \mathrm{FVC}>0.7$ ). COPDGene is a longitudinal, observational, and multicenter study investigating underlying genetic determinants of COPD (Clinicaltrials.gov identifier NCT00608764). DLCO was measured with EasyOne Pro (serial number 500633, ndd Medizintechnik AG, Zurich, Switzerland). Percent predicted values and $\mathrm{z}$ scores were calculated from raw values using Global Lung Function Initiative (GLI) equations. We processed inspiratory and expiratory $\mathrm{CT}$ images by parametric response mapping (PRM), a novel CT biomarker technique, using Imbio Lung Density Analysis dynamic

(c) The Author(s). 2019 Open Access This article is distributed under the terms of the Creative Commons Attribution 4.0 International License (http://creativecommons.org/licenses/by/4.0/), which permits unrestricted use, distribution, and 
image registration software (Minneapolis, $\mathrm{MN}$ ) to quantify areas of emphysema (PRM $\left.{ }^{\mathrm{Emph}}\right)$ and areas of nonemphysematous gas trapping recently determined to be SAD $\left(\mathrm{PRM}^{\mathrm{fSAD}}\right)$ [5]. Multivariable regression models for DLCO GLI z-score were created to determine the relative contribution of $\mathrm{PRM}^{\mathrm{Emph}}$ and $\mathrm{PRM}^{\mathrm{fSAD}}$, additionally adjusted for age, sex, and current smoking.

\section{Results}

Participant mean age at time of measurement was 67.0 years. With increasing GOLD stage, DLCO \% predicted fell and $\mathrm{z}$-scores became more negative, while PRM ${ }^{\mathrm{Emph}}$ and $\mathrm{PRM}^{\mathrm{fSAD}}$ rose. These characteristics, as well as other subject characteristics, are shown in Table 1.

In a multivariable regression model examining nonobstructed ever-smokers, PRM ${ }^{\mathrm{fSAD}}(\beta=-0.03, p<0.001)$ and $\mathrm{PRM}^{\mathrm{Emph}}(\beta=-0.04, p=0.03)$ were associated with lower DLCO GLI z-score. For clinical interpretation, on average a non-obstructed individual with $10 \%$ higher $\mathrm{PRM}^{\mathrm{fSAD}}$ would be predicted to have $3.1 \%$ lower DLCO $\%$ predicted. In GOLD $1-2$, both $\mathrm{PRM}^{\mathrm{fSAD}}(\beta=-0.02$, $p=0.004)$ and PRM ${ }^{\mathrm{Emph}}(\beta=-.10, p<0.008)$ were associated with lower DLCO GLI z-score. Among GOLD 34 , $\mathrm{PRM}^{\text {Emph }}(\beta=-0.11, p<0.001)$ but not $\mathrm{PRM}^{\mathrm{fSAD}}$ $(p=0.69)$ was associated with lower GLI z-score.

Table 1 Baseline demographics

\begin{tabular}{|c|c|c|c|}
\hline & \multicolumn{3}{|c|}{ GOLD spirometry grade } \\
\hline & $0(n=957)$ & $1-2(n=584)$ & $3-4(n=305)$ \\
\hline Age, yr & 63.5 & 68.3 & 68.9 \\
\hline Sex, n (\% female) & $512(54 \%)$ & $249(42 \%)$ & $130(43 \%)$ \\
\hline BMI $\left(\mathrm{kg} / \mathrm{m}^{2}\right)$ & 28.9 & 27.9 & 27.5 \\
\hline Smoking pack-years & 37.1 & 49.8 & 55.0 \\
\hline Current smokers, n (\%) & $344(36 \%)$ & $212(36 \%)$ & $61(20 \%)$ \\
\hline Exacerbations in prior year & 0.11 & 0.32 & 0.71 \\
\hline $\mathrm{FEV}_{1}, \mathrm{~L}$ & 2.73 & 2.03 & 0.98 \\
\hline $\mathrm{FEV}_{1} \%$ predicted & 98.4 & 73.3 & 36.4 \\
\hline FVC, L & 3.49 & 3.39 & 2.43 \\
\hline FVC \% predicted & 96.1 & 91.7 & 67.2 \\
\hline $\mathrm{FEV}_{1} / \mathrm{FVC}$ & 0.78 & 0.60 & 0.41 \\
\hline DLCO, \% predicted & $90.1 \%$ & $74.2 \%$ & $51.2 \%$ \\
\hline DLCO, GLI z-score & -0.73 & -1.91 & -4.08 \\
\hline $\mathrm{PRM}^{\mathrm{fSAD}}$ & $10.0 \%$ & $21.0 \%$ & $34.6 \%$ \\
\hline $\mathrm{PRM}^{\mathrm{Emph}}$ & $1.8 \%$ & $6.5 \%$ & $18.3 \%$ \\
\hline
\end{tabular}

All values expressed as mean except categorical variables expressed as $n(\%)$ $B M I$ body mass index, $F E V_{1}$ forced expiratory volume in $1 \mathrm{~s}, F V C$ forced vital capacity, DLCO single breath carbon monoxide diffusing capacity of the lung, GLI Global Lung Function Initiative, PRM parametric response mapping, $f S A D$ functional small airways disease, Emph emphysema

\section{Discussion}

Our analysis of a sizeable group of current and former smokers indicate that in those without airflow obstruction and in individuals with mild to moderate COPD, small airways disease defined by PRM is associated with significant gas exchange abnormalities. These data build on and extend recent work to elucidate the nature of small airway abnormality in COPD. The PRM ${ }^{\mathrm{fSAD}}$ metric has been associated with more rapid lung function decline, even among individuals with emphysema [6]. Using longitudinal image registration, we previously showed that voxels identified as $\mathrm{PRM}^{\mathrm{fSAD}}$ radiographically precede development of CT-determined emphysema in those same voxels [7]. Importantly, we recently demonstrated with severe COPD human lung tissue that $\mathrm{PRM}^{\mathrm{fSAD}}$ metric corresponds to pathologic abnormality, including decreased circularity, decreased luminal area, and complete obstruction of terminal bronchioles $(\mathrm{TBr})$, whereas $\mathrm{PRM}^{\mathrm{Emph}}$ is significantly associated with decreased alveolar surface area [5].

However, the pathology identified by $\mathrm{PRM}^{\mathrm{fSAD}}$ in milder disease is unknown. Given the totality of data generated to date, $\mathrm{PRM}^{\mathrm{fSAD}}$ is also likely associated with $\mathrm{TBr}$ pathology in mild COPD, but the extent to which it may also identify early alveolar destruction must be determined.

The association we show here between $\mathrm{PRM}^{\mathrm{fSAD}}$ and reduced DLCO suggests that the fSAD metric may detect lung beginning to transition from bronchiolar pathology to emphysema.

Support for this possibility comes from McDonough et al., who used micro-CT to analyze lung tissue samples in severe COPD. By identifying significantly reduced $\mathrm{TBr}$ numbers in areas of lung without visible emphysema evidence, they inferred that narrowing and loss of $\mathrm{TBr}$ precedes emphysema [2]. However, a linear relationship between loss of $\mathrm{TBr}$ and increase in mean linear intercept (a measure of mean free distance in the air spaces) was seen. Most recently, Koo et al. examined lung tissue from GOLD 1-2 stage disease and again found significant reductions in number of $\mathrm{TBr}$ and transitional bronchioles in lung areas without visible emphysema [3]. Collectively, these results support SAD as one precursor of emphysema.

DLCO is well-established to correlate with CTdetermined emphysema [4], but it may also be decreased in mild COPD, where emphysema is not detectable by CT imaging. Active smokers with normal spirometry but low DLCO had increased rate of progression to GOLDdefined COPD, compared to smokers with normal spirometry and normal DLCO, implying that isolated decreased DLCO is a risk factor for airflow obstruction in otherwise healthy subjects [8]. Ex-smokers with normal CTs and no airflow obstruction but with low DLCO had 
significantly worse symptoms and exercise capacity as well as greater regional lung destruction, as measured by hyperpolarized ${ }^{3} \mathrm{He}$ MRI apparent diffusion coefficients [9]. In 10 patients with severe SAD, defined by severe expiratory airflow limitation with mild CT-detected emphysema, Gelb et al. found DLCO reductions; however, they proposed that this was possibly due to uneven gas sampling from lung units with differing expiratory time constants, a function of SAD rather than lung parenchymal destruction [10].

Our current analysis of ever-smokers without obstruction and with GOLD 1-2 COPD showed that PRM ${ }^{\mathrm{fSAD}}$ correlates with low DLCO, suggesting $\mathrm{PRM}^{\mathrm{fSAD}}$ might detect airways transitioning to early emphysema with resulting impaired gas exchange. Although COPD is a heterogonous disease, it is possible that a significant portion of small airways disease patients will progress to emphysema. Therefore, in addition to already describing correlations between tissue pathology and $\mathrm{PRM}^{\mathrm{fSAD}}$ in severe disease, similar studies in milder disease are also needed to confirm the pathology type identified in this population, who may be more amenable to therapeutic intervention that may halt progression to emphysema.

\section{Abbreviations}

CT: Computed tomography; DLCO: Diffusing capacity of the lung; fSAD: Functional small airways disease; GLI: Global Lung Function Initiative;

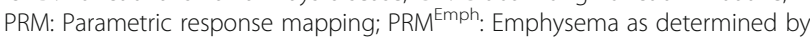
PRM; PRM ${ }^{\text {FAD }}$ : Small airways disease as determined by PRM; SAD: Small airways disease; TBr: Terminal bronchioles

\section{Acknowledgements}

Not applicable.

\section{Authors' contributions}

Concept and design: MKH, RNC. Data analysis: MKH. Data interpretation: MKH, RNC. Manuscript drafting and editing: all authors (RNC, CRH, CJG, EAK, DAL, MCM, RC, NRM, BJM, FJM, WWL, JLC, MKH). Final approval of the manuscript: all authors (RNC, CRH, CJG, EAK, DAL, MCM, RC, NRM, BJM, FJM, WWL, JLC, MKH)

\section{Funding}

This work was supported by NHLBI U01 HL089897 and U01 HL089856. The COPDGene study (NCT00608764) is also supported by the COPD Foundation through contributions made to an Industry Advisory Committee comprised of AstraZeneca, Boehringer-Ingelheim, GlaxoSmithKline, Novartis, and Sunovion. Dr. Han is supported by NHLBI K24 HL138188. Dr. Curtis is supported by 101 CX000911 from the Department of Veterans Affairs.

\section{Availability of data and materials}

The datasets used and analyzed during the current study are available from the corresponding author on reasonable request.

\section{Ethics approval and consent to participate}

This research letter presents an analysis of the COPDGene study registered on Clinicaltrials.gov (NCT00608764). The protocol was reviewed and approved by the Institutional Review Boards for each participating center (National Jewish Health, HS-1883a; Brigham and Women's Hospital, 2007-P000554/2; Baylor College of Medicine, H-22209; Michael E. DeBakey VAMC, $\mathrm{H}^{-22202}$; Columbia University Medical Center, IRB-AAAC9324; Duke University Medical Center, Pro00004464; Johns Hopkins University, NA_00011524; Los Angeles Biomedical Research Institute, 12756-01; Morehouse School of Medicine, 07-1029; Temple University, 11369; University of Alabama at Birmingham, FO70712014; University of California, San Diego, 070876; University of lowa, 200710717; Ann Arbor VA, PCC 2008-110732; University of Minnesota, 0801 M24949; University of Pittsburgh, RO07120059; University of Texas Health Sciences Center at San Antonio, HSC20070644H; Health Partners Research Foundation, 07-127; University of Michigan, HUM00014973; Minneapolis VA Medical Center, 4128-A; Fallon Clinic, 1143).

\section{Consent for publication}

Not applicable.

\section{Competing interests}

RNC, EAK, NM, RC, WWL - none.

$\mathrm{CRH}$ - An employee of and has stock options in Imbio LLC, which has

licensed the PRM technology for use as FDA-approved commercial software.

DAL - Reports grants from NHLBI and has a pending patent entitled Systems and Methods for Classifying Severity of COPD.

CJG - Is co-inventor of PRM, which is licensed to Imbio by the University of Michigan.

MM - Reports other support from UpToDate.

BM - Reports funding from the NHLBI for the COPDGene study; grants and medical advisory boards from Boehringer Ingelheim, GlaxoSmithKline,

AstraZeneca, and Sunovian; personal fees for DSMB from Spiration and Shire/ Baxalta; CME personal fees from WebMD, National Jewish.

Health, American College of Chest Physicians, Projects in Knowledge, Hybrid Communications, SPIRE Learning, Ultimate Medical Academy, Catamount Medical, Eastern Pulmonary Society, Catamount Medical Communications Medscape, Eastern VA Medical Center, Academy Continued Healthcare Learning, and Mt. Sinai Medical Center; royalties from Up-To-Date; medical advisory boards from Novartis, Phillips, Third Pole, Science 24/7, and Vernoa; and grants from Pearl.

FJM - Receives personal fees and non-financial support from American College of Chest Physicians, AstraZeneca, Boehringer Ingelheim, ConCert, Genentech, GlaxoSmithKline, Inova Fairfax Health System, Miller Communications, National Association for Continuing Education, Novartis, Pearl Pharmaceuticals, PeerView Communications, Prime Communications Puerto Rican Respiratory Society, Chiesi, Sunovion, Theravance, Physicians Education Resource, Canadian Respiratory Network, Teva, and Dartmouth. Receives non-financial support from ProterrixBio, Gilead, Nitto, Potomac, University of Alabama Birmingham, and Zambon. Receives personal fees from Columbia University, Integritas, MD Magazine, Methodist Hospital Brooklyn, New York University, UpToDate, WebMD/MedScape, Western Connecticut Health Network, Patara/Respivant, PlatformIQ, American Thoracic Society, Rockpointe, Rare Disease Healthcare Communications, and France Foundation. He receives grants from the $\mathrm{NIH}$. He also receives other support from Afferent/ Merck, Biogen, Veracyte, Prometic, Bayer, Bridge Biotherapeutics, and ProMedior.

JLC - Reports grants from NIH/NHLBI, NIH/NIAID, the Department of Veterans Affairs, and the Department of Defense.

$\mathrm{MKH}$ - Reports grants from NIH, personal fees from BI, GSK, AZ, and Mylan. She also receives other support from Novartis and Sunovion.

\section{Author details}

${ }^{1}$ Division of Pulmonary, Allergy and Critical Care, University of Pennsylvania, 839 West Gates Building, Philadelphia, PA 19104, USA. ${ }^{2}$ Imbio, LLC, Minneapolis, MN, USA. ${ }^{3}$ Department of Radiology, University of Michigan, Ann Arbor, MI, USA. ${ }^{4}$ Department of Radiology, National Jewish Health, Denver, CO, USA. ${ }^{5}$ Division of Pulmonary \& Critical Care Medicine, Johns Hopkins Medicine, Baltimore, MD, USA. ${ }^{6}$ Rehabilitation Clinical Trials Center, Los Angeles Biomedical Research Institute at Harbor-UCLA Medical Center, Torrance, CA, USA. 'Division of Pulmonary, Allergy and Critical Care Medicine, Duke University, Durham, NC, USA. ${ }^{8}$ Division of Pulmonary, Critical Care, and Sleep Medicine, National Jewish, Denver, CO, USA. ${ }^{9}$ Division of Pulmonary \& Critical Care Medicine, Weill Cornell Medical College, New York, NY, USA.

${ }^{10}$ Division of Pulmonary \& Critical Care Medicine, University of Michigan, Ann Arbor, MI, USA. " VA Ann Arbor Healthcare System, Ann Arbor, MI, USA.

Received: 21 September 2019 Accepted: 8 November 2019

Published online: 02 December 2019

\section{References}

1. Hogg JC, Macklem PT, Thurlbeck WM. Site and nature of airway obstruction in chronic obstructive lung disease. N Engl J Med. 1968;278:1355-60. 
2. McDonough JE, Yuan R, Suzuki M, Seyednejad N, Elliott WM, Sanchez PG, Wright AC, Gefter WB, Litzky L, Coxson HO, et al. Small-airway obstruction and emphysema in chronic obstructive pulmonary disease. N Engl J Med. 2011:365:1567-75.

3. Koo HK, Vasilescu DM, Booth S, Hsieh A, Katsamenis OL, Fishbane N, Elliott WM, Kirby M, Lackie P, Sinclair I, et al. Small airways disease in mild and moderate chronic obstructive pulmonary disease: a cross-sectional study. Lancet Respir Med. 2018;6:591-602.

4. D'Anna SE, Asnaghi R, Caramori G, Appendini L, Rizzo M, Cavallaro C, Marino G, Cappello F, Balbi B, Di Stefano A. High-resolution computed tomography quantitation of emphysema is correlated with selected lung function values in stable COPD. Respiration. 2012;83:383-90.

5. Vasilescu DM, Martinez FJ, Marchetti N, Galban CJ, Hatt C, Meldrum CA, Dass C, Tanabe N, Reddy RM, Lagstein A, et al. Non-invasive imaging biomarker identifies small airway damage in severe COPD. Am J Respir Crit Care Med. 2019;200:575-81.

6. Bhatt SP, Soler X, Wang X, Murray S, Anzueto AR, Beaty TH, Boriek AM, Casaburi R, Criner GJ, Diaz AA, et al. Association between functional small airway disease and FEV1 decline in chronic obstructive pulmonary disease. Am J Respir Crit Care Med. 2016;194:178-84.

7. Labaki WW, Gu T, Murray S, Hatt CR, Galban CJ, Ross BD, Martinez CH, Curtis $J \mathrm{~L}$, Hoffman EA, Pompe E, et al. Voxel-wise longitudinal parametric response mapping analysis of chest computed tomography in smokers. Acad Radiol. 2019;26:306-12

8. Harvey B-G, Strulovici-Barel Y, Kaner RJ, Sanders A, Vincent TL, Mezey JG, Crystal RG. Risk of COPD with obstruction in active smokers with normal spirometry and reduced diffusion capacity. Eur Respir J. 2015;46:1589-97.

9. Kirby M, Owrangi A, Svenningsen S, Wheatley A, Coxson HO, Paterson NAM, McCormack DG, Parraga G. On the role of abnormal $D L_{c o}$ in ex-smokers without airflow limitation: symptoms, exercise capacity and hyperpolarised helium-3 MRI. Thorax. 2013;68:752-9.

10. Gelb AF, Zamel N, Hogg JC, Müller NL, Scheiin MJ. Pseudophysiologic emphysema resulting from severe small-airways disease. Am J Respir Crit Care Med. 1998;158:815-9.

\section{Publisher's Note}

Springer Nature remains neutral with regard to jurisdictional claims in published maps and institutional affiliations.

Ready to submit your research? Choose BMC and benefit from:

- fast, convenient online submission

- thorough peer review by experienced researchers in your field

- rapid publication on acceptance

- support for research data, including large and complex data types

- gold Open Access which fosters wider collaboration and increased citations

- maximum visibility for your research: over $100 \mathrm{M}$ website views per year

At $\mathrm{BMC}$, research is always in progress.

Learn more biomedcentral.com/submissions 